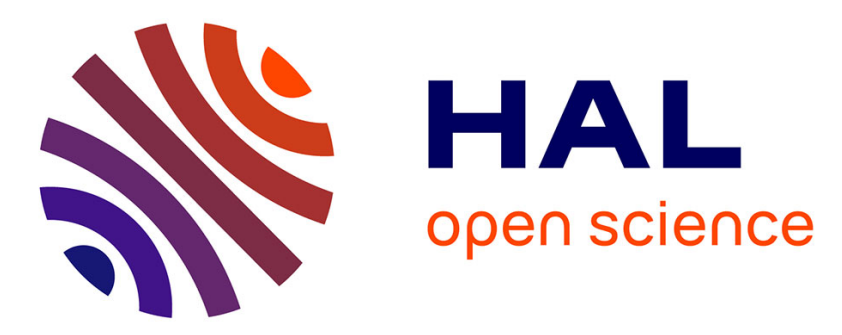

\title{
Index de contenu traumatique au Rorschach (TCI) et diagnostic de l'abus sexuel chez l'enfant
}

Sara de Michèle, Bernard Kabuth, Orlane Plun, Loïc Le Moal, Fanny Laurent, Elena Vandelet, Cécile Prudent, Claude de Tychey

\section{To cite this version:}

Sara de Michèle, Bernard Kabuth, Orlane Plun, Loïc Le Moal, Fanny Laurent, et al.. Index de contenu traumatique au Rorschach (TCI) et diagnostic de l'abus sexuel chez l'enfant. Annales MédicoPsychologiques, Revue Psychiatrique, 2019, 177 (6), pp.512-516. 10.1016/j.amp.2018.03.002 . hal02313285

\section{HAL Id: hal-02313285 \\ https://hal.univ-lorraine.fr/hal-02313285}

Submitted on 25 Oct 2021

HAL is a multi-disciplinary open access archive for the deposit and dissemination of scientific research documents, whether they are published or not. The documents may come from teaching and research institutions in France or abroad, or from public or private research centers.
L'archive ouverte pluridisciplinaire HAL, est destinée au dépôt et à la diffusion de documents scientifiques de niveau recherche, publiés ou non, émanant des établissements d'enseignement et de recherche français ou étrangers, des laboratoires publics ou privés.

\section{(ㅇ)(1) $\$$}

Distributed under a Creative Commons Attribution - NonCommerciall 4.0 International 


\section{Mémoire}

Index de contenu traumatique au Rorschach (TCI) et diagnostic de l'abus sexuel chez l'enfant

\section{Rorschach trauma content index (TCI) and child sexual abuse diagnostic}

Claude De Tychey ${ }^{a}$, Sara De Michele ${ }^{b}$, Bernard Kabuth ${ }^{c}$, Orlane Plun ${ }^{d}$, Loïc Le Moal ${ }^{\text {, }}$, Fanny Laurent ${ }^{\mathrm{f}}$, Elena Vandelet ${ }^{\mathrm{g}}$, Cecile Prudent ${ }^{\mathrm{h}}$

a) Claude de Tychey, Université de Lorraine, Campus SHS, Nancy2, Nancy, France

b) Sara de Michele GR3 P (Groupe de Recherche en Psychopathologie clinique Projectiveaxe Prévention) Laboratoire Interpsy (EA 4432), Université de Loraine, Nancy, France

c) Bernard Kabuth, Ph-PU Chef du service de pédopsychiatrie .Hôpital, Laboratoire Interpsy (EA 4432), Université de Lorraine, Nancy, France

d) Orlane Plun, Psychologue clinicienne au service de pédopsychia, Laboratoire Interpsy (EA 4432), Université de Lorraine, Nancy, France

e) Loïc Le Moal, Psychologue clinicien au service de pédopsychiat, Laboratoire Interpsy (EA 4432), Université de Lorraine, Nancy, France

f) Fanny Laurent, GR3 P (Groupe de Recherche en Psychopathologie clinique Projective-axe Prévention) Laboratoire Interpsy (EA 4432), Université de Lorraine, Nancy, France

g) Elena Vandelet, Psychologue clinicienne, doctorante au GR3 P (Gro, GR3 P) (Groupe de Recherche en Psychopathologie clinique Projective-axe Prévention) Laboratoire Interpsy (EA 4432), Université de Lorraine, Nancy, France

h) Cécile Prudent, Psychologue clinicienne, doctorante au GR 3P (Grou, Laboratoire Interpsy EA N ${ }^{\circ} 4432$ ), université de Lorraine-Campus SHS de Nancy 2, Nancy, France

\section{Texte reçu le 20 octobre 2017 ; accepté le 11 février 2018}

\section{Résumé}

Objectifs. - Présenter d'abord au clinicien expert une échelle construite à partir du test Rorschach, appelée TCI (index de contenu traumatique) par les psychologues américains codifiant le test selon la méthode Exner ou R-PAS, qui n'a jamais fait l'objet d'une publication en langue française. Montrer ensuite au lecteur que cet index est facilement transposable dans le système de cotation Rorschach de l'École de Paris et mettre sa validité à l'épreuve des faits dans le diagnostic de l'abus sexuel chez l'enfant. 
Méthode. - Cinquante enfants dont les dossiers d'expertise psychologique pour abus sexuel ont été rééxaminés par deux experts ayant conclu à une forte probabilité de la véracité de cet abus. L'index Rorschach de contenu traumatique TCI (Armstrong-Loewenstein, 1990 traduit dans le système de cotation de l'École de Paris pour l'étude portant sur ces 50 sujets âgés de 5 à 12 ans) a été comparé par deux praticiens, en cotation à double aveugle, avec celui de 50 enfants tout venants n'ayant jamais consulté. Les échantillons ont été appariés quant au NSE parental.

Résultats. - Le seuil de différenciation relatif à un index égal ou supérieur 0,25 (soit $25 \%$ au moins de réponses renvoyant à un contenu traumatique) proposé dans la validation anglosaxonne permet de différencier statistiquement les deux groupes de notre population française en identifiant $72 \%$ des enfants du groupe clinique contre seulement $6 \%$ de faux-positifs dans le groupe témoin.

Conclusion. - L'index TCI est pertinent dans la clinique de l'expertise psychologique. Des pistes de recherche sont suggérées.

Mots clés : Agression sexuelle; Diagnostic ; $\square$ Enfant ; Étude de cohorte; $\square \square$ Expertise judiciaire ; $\square$ Expertise psychologique ; Test Rorschach

\section{Abstract}

Objectives. - The article has two aims: first to present to French clinicians working in forensic field a translation of an index built and validated using Rorschach Inkblot method by Armstrong \& Loewenstein (1990), called Trauma Content Index (TCI). TCI was codified inside Comprehensive System and integrated in recent R-PAS. The Rorschach indicators that constitute TCI, namely responses related to Blood, Anatomy, Sex, aggressive movement and morbid content can be easily translated in French Parisian School system. For us, these indicators are highly relevant because they refer, according to our conception, to the attack of the image of the body and the representation of self which are the first indicators to be degraded in case of sexual abuse. Second objective is to study if American validated TCI cut off to identify sexual abuse has the same usefulness when applied to a cohort of French children suspected of sexual abuse than in American sample and using Parisian School Rorschach codifying system.

Methods. - We compared TCI of 50 children aged from 5 to 12 evaluated with Rorschach Inkblot A methodology in double-blinded-scoring where the two experts were both convinced of probability child sexual abuse diagnosis. We have obtained a high fidelity score of 
interrater agreement (0.92). These data were compared to TCI index of 50 children matched control group with samples stratified according to socio-economic level of parents as well as age. Chi square method was used to compare the two groups.

Results. - A TCI cut off of 0,25 allows us to differentiate statistically the two groups (Chi2: $19,01 ; \mathrm{p}<.001)$. Such a cut off enables us to identify $72 \%$ of the clinical group and only $6 \%$ of the control group as false positives, showing the interest of TCI index applied to children suspected of sexual abuse. We agree with point of view issued from Kamphuis-Kugeares \& Finn research (2000) upon adults in their own samples $(n=100)$. That study compared Rorschach records of non-dissociative outpatients with histories of (a) definite sexual abuse, (b) suspected but unconfirmed sexual abuse or (c) non-sexual abuse. They conclude that, although TCI was strongly associated with the presence and severity of sexual abuse, the TCI is one factor among many that can be used to assess the validity of clients' claims of past sexual abuse. French and south America studies on children with projective tests (Condamin 2006, Mazoyer-Roques, 2014, Scortegagna \& de Villemor-Amoral 2012) are more qualitative oriented than Anglo-saxons researches (Leifer et al. 1991, Kikuchi et al. 2010), but they end up at the same conclusion. That is the reason why we compared our two groups with as second projective tool: the ant's tales test (Royer 1978, de Tychey 2010) used to study child's body image whose usefulness was recently suggested to identify child sexual abuse (Kabuth et al 2017). Join use of TCI and indicators issued from that tool allow us to identify $94 \%$ (instead of $72 \%$ with TCI single use) of our clinical group

Conclusion. - TCI and projective tools are useful in forensic field to undertake child sexual abuse diagnosis. It would be important for future research to make comparative studies to approach TCI cut off scores derived from subjects confronted to various post traumatic situations and their psychiatric disorders forms. So it would perhaps possible to highlight specific cut off values to child sexual abuse diagnosis.

Keywords: Child; Cohort study; Diagnostic; Judicial expertise; Psychological expertise; Rorschach test; Sexual assault

\section{Introduction, objectifs}

L'approche clinique de l'abus sexuel chez l'enfant reste, malgré la pléthore des recherches qu'elle a suscitée, une entreprise malaisée. Les travaux scientifiques qui ont cherché à évaluer sa fréquence aboutissent à des taux de prévalence de sa survenue 
particulièrement disparates au vu des recherches internationales comparatives entreprises [11,4] en raison des nombreux biais méthodologiques qui ont saturé la plupart de ces recherches [9].

Les mêmes discordances peuvent s'observer, le procès d'Outreau est là pour nous le rappeler, lorsqu'il s'agit d'établir avec fiabilité ce difficile diagnostic d'abus sexuel chez l'enfant dans le cadre d'une expertise judiciaire ou d'une consultation clinique standard. S'appuyer sur la seule parole consciente de l'enfant ou de l'auteur présumé de l'agression n'est nullement suffisant. En effet, celle de l'enfant peut être déformée par la pression des adultes tutélaires et celle de l'auteur présumé de l'agression peut être induite par la culpabilité, les conditions de la garde à vue et les mécanismes de défense intrapsychiques qu'il peut mettre en œuvre. Ce constat a conduit les psychologues cliniciens à essayer de s'appuyer sur des épreuves projectives, principalement celle qui est la mieux validée à leurs yeux, à savoir le test de Rorschach, pour tenter d'asseoir le diagnostic d'abus sexuel. L'utilisation de cet outil en France est le plus souvent envisagée alors dans une perspective qualitative. Elle suggère son intérêt pour légitimer le diagnostic d'abus sexuel, soit à partir de vignettes cliniques d'enfants certes édifiantes mais ne permettant aucune généralisation des marqueurs mis en évidence [3,21] soit à partir d'un échantillon un peu plus important : 26 fillettes pour Richelle [25] ou plus récemment l'étude rétrospective cette fois d'une quinzaine d'adultes abusés durant l'enfance [23] .On retrouve la même orientation dans les travaux sud-américains, qu'ils soient argentins [12] ou brésiliens [30]. Malheureusement, on regrettera qu'aucune de ces études françaises et sud-américaines, outre la taille restreinte des effectifs, n'ait été conduite dans une perspective comparative avec un groupe contrôle.

Un tel double reproche ne peut cependant être adressé aux cliniciens anglo-saxons travaillant dans ce champ avec le test de Rorschach et un système de codification du test différent de celui de l'École de Paris [2,24,26] qui a évolué dans le temps avec d'abord le Comprehensive System (C.S.) d'Exner [10] puis le Rorschach Personality Assessment System appelé R-PAS [22]. En effet, la clinique anglo-saxonne a montré, à partir d'études quantitatives comparatives, tout l'intérêt du test de Rorschach pour le diagnostic d'abus sexuel $[1,17,19,20]$ et encore plus généralement pour le diagnostic de traumatisme [31] ou l'exposition à des situations de violence [18]. Au centre de la plupart de ces recherches se trouve une échelle de traumatisme construite à partir des contenus projetés au test de Rorschach appelée «Trauma content » par Armstrong-Loewenstein [1] puis rebaptisée T.C.I. «trauma content index dans les travaux empiriques que nous venons d'évoquer. 
Notre premier objectif sera d'effectuer une traduction de cette échelle qui n'a à notre connaissance jamais été présentée aux cliniciens de langue française utilisateurs de la codification de l'École de Paris. Certains pourront trouver ce choix à risques car la méthode de cotation américaine du test de Rorschach pour un certain nombre de ses indicateurs est différente de la méthode de l'École de Paris et non transposable, en particulier pour certains indices exigeant un calcul statistique sophistiqué. Par contre, l'ensemble des contenus composant le TCI (Trauma content Index) présente l'avantage de pouvoir être relativement facilement transposable dans la méthode de codification de l'École de Paris, ce qui nous semble de nature à permettre de rapprocher les deux systèmes de codification plutôt qu'à chercher à les opposer.

Notre second objectif sera de mettre à l'épreuve pour la première fois la validité diagnostique du TCI sur une population d'enfants français dont l'expertise judiciaire a été conclue par la conviction, pour chaque expert, de la haute probabilité de l'abus sexuel allégué par ces enfants. Ces enfants seront comparés à un groupe témoin apparié sur le plan du NSE des parents et situé dans la même tranche d'âge, 5-12ans.

Avant de préciser les choix méthodologiques qui ont été les nôtres, nous souhaitons présenter rapidement au lecteur le mode de calcul du TCI et à partir des contenus passés en revue, réfléchir à partir d'un modèle psychodynamique, au rationnel théorique qui sous-tend sa construction, ce que n'ont absolument pas fait les créateurs et utilisateurs anglo-saxons de cette «échelle » élaborée par eux dans une perspective strictement empirique. L'index de contenus traumatiques (TCI) se calcule en sommant dans la version américaine les contenus $\mathrm{B} 1+\mathrm{An}+\mathrm{Sex}+\mathrm{AG}($ devenu AGM dans le R-PAS) + MOR et en le divisant par R qui est le nombre total de réponses au test. Un score égal ou supérieur à 0,25 (correspondant en fait à $25 \%$ de contenus problématiques par rapport au total des réponses du sujet) est posé dans les recherches anglo-saxonnes comme valide pour identifier les enfants victimes d'abus sexuel ou d'adultes victimes d'abus sexuel dans l'enfance. La traduction de ces indicateurs dans le système de l'École de Paris est relativement aisée. Bl (Blood) correspond aux contenus faisant référence au sang $(\mathrm{Sg})$, An (Anatomy) aux contenus anatomique (Anat), Sex aux réponses sexuelles, AG (devenu AGM) correspondant dans l'École de Paris aux différents types de réponses kinesthésiques mettant en scène un mouvement agressif. La transposition du dernier indicateur MOR (renvoyant chez les Anglo-Saxons aux différents types de contenus morbides et/ou comprenant une connotation dysphorique) est un peu plus complexe car renvoyant à plusieurs dimensions dans le système de cotation française. Dans notre codification française, il faudrait coter MOR pour toute réponse comportant une dimension de détérioration (que 
nous cotons habituellement dans la colonne qualitative par le terme de « défect») mais également pour toute réponse dysphorique comportant une tonalité explicite soit d'angoisse (tout réponse incluant le déterminant Clob dans notre cotation) soit de dépression (associée ou non au déterminant $C^{\prime}$ ).

Analysés sous l'angle de notre modèle théorique psychodynamique du trauma, les contenus composant le TCI semblent d'une grande cohérence. En effet, les réponses sang, anatomiques et sexuelles sont des réponses traduisant une attaque de l'image du corps et de la représentation de soi aisément compréhensible en cas d'abus sexuel. Elles témoignent d'une désymbolisation associée à l'effraction de la barrière de pare-excitation. Les réponses kinesthésiques comportant une valence agressive expriment habituellement une représentation de relation sur le mode de la menace ou du risque d'agression. Enfin au niveau des contenus morbides, le défect renvoie à la même expression de l'attaque de l'image du corps et de la représentation de soi tandis que la projection d'affects anxiogènes ou dépressifs est facilement de mise dans un contexte traumatique. Mais au-delà de sa cohérence théorique, le TCI offre-til des repères diagnostiques aussi valides sur une population d'enfants français que ceux enregistrés sur les populations anglo-saxonnes? Notre recherche va s'attacher à mettre à l'épreuve cette question sur le plan empirique.

\section{Méthodes}

\subsection{Critères de sélection du groupe clinique et du groupe témoin}

Les dossiers d'expertise judiciaire des 50 enfants victimes de sévices ont été réalisés par deux membres de l'équipe appartenant à une unité fonctionnelle d'accueil des enfants victimes de sévices. Le recrutement des 50 enfants du groupe témoin a été réalisé dans deux écoles de l'agglomération nancéenne après que les parents de ces enfants ont signé un formulaire de consentement éclairé et que l'administration scolaire a donné son accord pour que l'évaluation psychologique soit réalisée dans le cadre scolaire de ces enfants.

Après que l'ensemble des tests de Rorschach des deux groupes ont été anonymisés, la codification du test de Rorschach des enfants des deux groupes a été réalisée en double aveugle par deux autres membres de l'équipe de recherche avec l'accord du médecin-chef du service de l'unité fonctionnelle d'accueil des enfants victimes de sévices pour le groupe clinique. La fidélité inter-coteur de cotation de l'index TCI s'est avérée excellente puisque la corrélation obtenue est de .92 . 
Le groupe clinique est composé de 50 enfants sans pathologie mentale âgés de 5 à 12 ans (âge moyen : 7 ans 9 mois). Il est composé de 39 filles et 11 garçons, ce qui le rend assez représentatif de la population générale des enfants victimes d'abus puisque le sex-ratio mis en évidence dans les études épidémiologiques suggèrent une fréquence d'occurrence de ce traumatisme habituellement trois à quatre fois plus fréquente chez les filles [3,11]. Le motif de la demande d'expertise judiciaire renvoyait à différents types d'abus (7 enfants étaient suspectés d'avoir subi des viols intrafamiliaux répétés, 3 un viol intrafamilial unique, 8 des viols extrafamiliaux répétés, 6 un viol extrafamilial unique, 6 des attouchements sexuels extrafamiliaux répétés, 12 des attouchements sexuels intrafamiliaux répétés, 4 un attouchement sexuel extrafamilial unique et 4 un attouchement sexuel intrafamilial unique).

Le groupe témoin est composé de composé de 50 enfants sans pathologie mentale, âgés de 5 à 12 ans dont l'âge moyen (9 ans 5 mois) est un peu plus élevé et sans antécédents d'hospitalisation pour une opération car nous avons souhaité contrôler l'impact de cette variable sur une atteinte potentielle de l'image du corps lors de l'investigation clinique projective. Il était composé de 27 filles et 23 garçons car nous souhaitions constituer un échantillon témoin se rapprochant davantage du sex-ratio de la population générale des enfants tout-venants.

\subsection{Outils utilisés}

Pour le groupe clinique qui avait fait l'objet d'un examen psychologique complet, nous avons, à partir des dossiers comprenant au niveau des épreuves projectives passées un test de Rorschach et le conte de la fourmi extrait du test des contes (Royer [27], de Tychey [8]), entrepris la codification de l'index de contenus traumatiques TCI selon la méthode de l'École de Paris afin de pouvoir répartir les enfants de part et d'autre du score seuil critique de 0,25 (correspondant pour rappel à $25 \%$ au moins des réponses au test à valence traumatique).

Pour le groupe témoin, outre l'entretien clinique anamnestique avec l'enfant et sa mère, nous avons entrepris la codification de l'index TCI du test de Rorschach de façon similaire à celle du groupe clinique par deux membres de l'équipe autres que ceux qui avaient procédé au recueil des données.

\subsection{Hypothèse de travail}


Nous avons posé l'hypothèse que l'index TCI devait permettre, en prenant un score seuil de 0,25, de différencier significativement les deux échantillons et d'identifier un nombre important d'enfants du groupe clinique.

\subsection{Type de traitement statistique des données}

Les données des deux groupes ont été comparées par la méthode du chi 2.

\section{Résultats}

Notre hypothèse de travail est confirmée sur le plan empirique. En effet dans le groupe clinique, 36 enfants sur 50 (72\%) dont l'abus sexuel est probable ont un score TCI supérieur ou égal au seuil limite de 0,25 (2,5\%) contre seulement 3 enfants sur 50 (6\%) dans le groupe contrôle. La différence est hautement significative sur le plan statistique (Chi $2=19,16$, $\mathrm{p}<$.001). À un niveau très général, le TCI moyen du groupe clinique est de 0,37 et celui du groupe témoin de 0,10 . Nous allons illustrer ce résultat par une double vignette clinique. Melle $\mathrm{O}$ est une petite fille âgée de 8 ans et 2 mois. Elle est fille unique. Son père ne s'est pas investi auprès d'elle. Ses parents ont divorcé lorsqu'elle avait 5 ans. En ce qui concerne les faits de cette expertise psychologique, elle a subit un viol extrafamilial unique durant un séjour en colonie de vacances par un adolescent. Suite au viol, elle a ressenti des douleurs durant quelques jours. Elle tremblait et avait peur en parlant de l'acte. Elle avait quelques connaissances sur la différence des sexes mais pas sur la sexualité. Après, cet événement, elle développe des troubles du sommeil, des réveils nocturnes, une énurésie secondaire, son comportement alimentaire se modifie (mange plus qu'avant). De plus, elle exprime un symptôme de stress post-traumatique sous forme de craintes de répétitions que les mêmes faits se produisent par le même auteur, de ruminations et réminiscences refoulées malgré les flashbacks récurrents, et une forte culpabilité. Le test de Rorschach comporte 26 réponses dont 11 correspondent à des contenus à valence traumatique entrant dans le calcul de l'index TCI. Elles sont constituées de sept réponses codifiées défect dans la cotation de l'École de Paris (« des tissus en morceaux », «Un petit bout d'araignée », «Un morceau de sapin », «Des bouts de peau», « des bouts de peau », « un monstre à deux têtes », « des morceaux de peau»), d'une réponse anatomique à valence sexuelle («des seins»), d'une réponse kinesthésique comportant une valence agressive («les bouts de tissus en train de s'arracher ») et de deux contenus comportant une dimension de détresse-dysphorie («Un monstre qui est 
tout noir » et «Des monstres »). Son score TCI est de 0,42. À l'opposé, Mlle M., une fillette du même âge, donne sur 17 réponses au test de Rorschach 3 contenus qui sont à coter sur l'index TCI : deux contenus comportant une dimension de dysphorie anxiogène (deux fois «un monstre ») et une réponse défect («un monstre sans tête »), ce qui aboutit pour elle à un score TCI de 0,17 .

\section{Discussion}

Les données comparées obtenues pour les deux groupes sont intéressantes. En effet, le pourcentage d'enfants du groupe clinique présentant un score TCI significatif (72\%) est intéressant et le pourcentage de faux positifs dans le groupe contrôle $(6 \%)$ est très faible. Il peut s'expliquer par le fait que ces enfants du groupe contrôle ont pu vivre au cours de leur trajectoire existentielle un autre traumatisme existentiel qu'un abus sexuel, ce que nos éléments informatifs anamnestiques les concernant, trop succincts, n'ont pas permis de vérifier. Le score TCI, quand il est élevé, apparaît comme un indicateur intéressant d'identification des enfants suspectés fortement d'abus sexuel, mais les $28 \%$ de faux négatifs enregistrés dans notre étude suggèrent qu'il ne peut être utilisé isolément à des fins diagnostiques. Ce qui rejoint les conclusions des auteurs anglo-saxons qui l'ont mis à l'épreuve dans leurs propres recherches. Khampuis-Kugeares \& Finn [17], qui l'ont testé sur trois types de populations : des personnes dont l'abus sexuel est avéré, d'autres où l'abus est suspecté, et enfin des personnes n'ayant jamais subi d'abus sexuel, ont remarqué que plus le degré de certitude de l'abus sexuel augmente et plus le TCI moyen augmente lui aussi. Ces auteurs notent qu'il faut bien prendre en compte le fait que cet indicateur présente une sensibilité à d'autres types de traumatismes que l'abus sexuel, et de ce fait qu'il ne peut être utilisé comme seul et unique indicateur pour prouver un abus sexuel. Ces auteurs anglosaxons prônent donc la convergence entre plusieurs outils diagnostiques. Nous suggérons également ce choix qui nous paraît très pertinent pour tout clinicien français engagé dans le champ de l'expertise judiciaire. Nous l'avons d'ailleurs également envisagé dans le cadre de notre recherche en nous appuyant sur un autre outil projectif dont l'utilité diagnostique apparaît indiscutable [16], le conte de la fourmi, qui permet d'approcher l'attaque et les avatars de l'image du corps de l'enfant ${ }^{1}$. Les enfants faisant l'objet d'un diagnostic d'abus sexuel fantasment à ce conte significativement plus souvent un trou d'entrée et/ou de sortie du

\footnotetext{
Le texte du conte de la fourmi se trouve en annexe.
} 
corps de l'enfant à valence régressive (sexuelle) ou un trou imaginaire. Ils font également beaucoup plus de confusions intérieur/extérieur au niveau du trajet interne de la fourmi. Ils projettent aussi beaucoup plus souvent un dénouement négatif, ce qui traduit l'attaque traumatique de l'image du corps. En mettant en convergence ce conte avec le TCI, le taux de faux négatifs dans notre groupe clinique (pour rappel,14 enfants au vu du seul TCI) passe de $28 \%$ à $6 \%$ (seuls 3 enfant sur 50 passent au travers de l'identification diagnostique). Au niveau diagnostique, l'évaluation de l'abus reste une tâche complexe qui a fait l'objet de revues pertinentes ces vingt dernières années, en particulier de la part de nos collègues cliniciens belges $[5,13,14,15]$. Nous partageons pleinement la position d'Emmanuel de Becker [6] qui souligne, à côté de l'entretien clinique, la nécessité d'une approche diagnostique pluridisciplinaire et, sur le plan du bilan de la sphère affective, le recours à des outils projectifs multiples. Pas seulement le test de Rorschach qui a été l'objet central de ce travail mais également le C.A.T., le T.A.T. ainsi que le test des contes, en plus des épreuves faisant appel au dessin dont l'utilité a été démontrée depuis de nombreuses années [7,28,29].

Nous restons conscients néanmoins des limites et des biais qui ont pu infiltrer notre propre investigation et l'index TCI que nous avons privilégié dans cette étude. En premier lieu, nous pensons qu'il faudrait augmenter la taille des deux échantillons français comparés et leur degré d'homogénéité. En effet, nous avons, pour constituer notre groupe clinique, retenu des enfants qui avaient subi soit un abus unique, soit des abus multiples de nature différente, commis soit par un proche soit par un membre extérieur à la famille. Or nous pouvons faire l'hypothèse, en suivant les constats antérieurs réalisés par d'autres cliniciens [13], qu'un viol répété commis par un membre de la famille a probablement un impact désorganisateur plus grand qu'un attouchement unique commis par un étranger qui suscitera immédiatement un étayage protecteur plus grand de la famille de l'enfant, et donc que les deux situations ne sont pas complètement comparables. Sur un autre plan, nous sommes partis pour sélectionner notre groupe clinique de la conviction des deux experts, chargés de l'expertise, de la véracité du dire de l'enfant. Nous pensons qu'une recherche ultérieure gagnerait à comparer les production projectives d'un groupe clinique d'enfants suspectés d'abus à celles d'un échantillon d'enfants dont l'abus serait médicalement prouvé, en contrôlant encore davantage que nous avons pu le faire dans notre propre recherche l'âge des enfants, l'âge au moment de l'abus et l'intervalle temporel entre le moment de l'abus et l'évaluation psychologique de l'enfant.

\section{Conclusion}


Cette recherche suggère l'intérêt du recours à une investigation clinique projective dans le cadre d'une expertise judiciaire relative à la détection de l'abus sexuel. L'indicateur TCI obtenu à partir du test de Rorschach reçoit sur notre propre population française une confirmation empirique de sa valeur diagnostique déjà suggérée par les travaux anglo-saxons. Il nous semblerait très judicieux, dans les recherches futures ayant recours à la codification du test selon la méthode de l'École de Paris, d'affiner sa valeur diagnostique différentielle de manière plus précise. En effet, cet indicateur, en l'état actuel et avec le sous-bassement théorique qu'on peut lui prêter, est construit pour être sensible non seulement à un traumatisme de nature sexuelle mais probablement à l'impact d'autres traumatismes que ceux de la sphère sexuelle. Dans ces conditions, il faudrait s'efforcer de comparer les distributions de scores TCI de sujets victimes d'abus à celles de sujets présentant par exemple un PTSD lié à un autre événement de vie et à celles d'autres groupes psychopathologiques dont on peut faire l'hypothèse qu'ils ont été confrontés à d'autres types de traumatismes au cours de leur trajectoire existentielle. L'objectif serait de voir si des valeurs spécifiques d'index TCI pourraient ou non être dégagées pour chacun de ces sous-groupes.

\section{Conflit d'intérêts : aucun}

\section{Références}

[1] Armstrong JG, Loewenstein RJ. Characteristics of patients with multiple personality and dissociative disorders on psychological testing. J Nervous Ment Disease 1990;178:448-54.

[2] Chabert C. La psychopathologie à travers le Rorschach. Paris: Dunod; 1987.

[3] Condamin C. Corps démembré, corps supplicié, corps massacré. Champ psy 2006;41:129-42.

[4] Cosentino CE. Sexual abuse of children: Prevalence, effects, and treatment. In Sechzer JA, Pfafflin SM, Denmark FL. Ed. Women and mental health, New York: New York Academy of Sciences; 1996. P. 45-65.

[5] De Becker E, Maertens MA. Le devenir de l'enfant victime de maltraitance sexuelle. Ann Med Psychol 2015;173:805-14.

[6] De Becker E, L'enfant victime d'abus sexuel et sa famille : évaluation et traitement. Vingt ans après. Ann Med Psychol (Paris) 2017 ;175,415-421. 
[7] de Tychey C, Cobai O. Le diagnostic des abus sexuels à travers le dessin : présentation de la grille diagnostique de Van Hutton. Pratiques Psychologiques 2000;4:13-32.

[8] de Tychey C. Test des contes et clinique infantile. Paris: InPress; 2010.

[9] de Tychey C, Laurent M, Lighezzolo-Alnot J, Garnier S, Vandelet E. Prevalence of Sexual Abuse in Childhood: Some Critical Methodological Reflections. J Child Sex Abuse 2015;24:401-11.

[10] Exner JE. The Rorschach: a comprehensive system. New-York: Wiley; 1986.

[11] Finkelhor D. The international epidemiology of child sexual abuse. Child Abuse \& Neglect 1994;18:409-17.

[12] Gravenhorst MC. Rorschach Psychodiagnosis of Psychic Trauma in Sexually Abused Children. Rorschachiana 2002; 25:77-86.

[13] Hayez Y. L'enfant victime d'abus sexuel et sa famille : évaluation et traitement. Paris: PUF; 1984.

[14] Hayez JY, de Becker E. L'enfant victime d'abus sexuel et sa famille. Évaluation et traitements. Paris: PUF; 1997.

[15] Hayez JY, de Becker E. La parole de l'enfant en souffrance : accueillir, évaluer et accompagner. Paris: Dunod; 2010.

[16] Kabuth B, Plun O, Le Moal L, Vandelet E, Prudent C, Laurent M, de Tychey C. Abus sexuel chez l'enfant, image du corps et conte de la fourmi. Revue québecoise de Psychologie 2018 ( à paraître).

[17] Kamphuis JH, Kugeares SL,Finn SE. Rorschach Correlates of Sexual Abuse: Trauma Content and Aggression Indexes. J. Pers. Assessm 2000;75:212-24.

[18] Kamphuis JH, Tuin N, Timmermans M, Punamäki RL. Extending the Rorschach Trauma Content Index and Aggression Indexes to Dream Narratives of Children Exposed to Enduring Violence: An Exploratory Study. J. Pers. Assessm 2008;90:578-84.

[19] Kikuchi K, Kikuchi Y, Horikawa Y, Horikawa K. Verification of Rorschach Indicators of Sexual Abuse. Yonago Acta medica 2010;53:53-8.

[20] Leifer M, Shapiro J, Martone M, Kassem M. Rorschach assessment of psychological functioning in sexually abuses girls. J Pers Assessm 1991;56:14-28.

[21] Mazoyer AV, Roques R. Mobilisation des processus psychiques chez des enfants victimes d'agression sexuelle. Contribution du Rorschach à la clinique du trauma. Bull Psychol 2014;532:331-48. 
[22] Meyer, GJ, Viglione, DJ, Mihura JL, Erard RE, Erdberg P. Rorschach Performance Assessment System: Administration, coding, interpretation, and technical manual. Toledo, OH: Rorschach Performance Assessment System, LLC; 2011.

[23] Moreno-Galan S, Masselin-Dubois A, François L. Sexually abused women's body image through the Rorschach test. Communication au Congrès international du Rorschach et des Méthodes Projectives, 2017 Jul 17-21; Paris.

[24] Rausch de Traubenberg N. La pratique du Rorschach. Paris: PUF; 1970.

[25] Richelle J. Abus sexuels : identité et identité sexuelle à travers le test de Rorschach. Bull Soc Rorschach \& Méthodes Projectives 1994;38:85-90.

[26] Richelle J, de Noose L, Malempré M, Debroux P. Manuel du test de Rorschach: approche formelle et psychodynamique. Bruxelles: De Boeck; 2009.

[27] Royer J. Le test des contes. Paris: E.A.P; 1978.

[28] Royer J. Que nous disent les dessins d'enfants ? Paris: Le Journal des psychologues; 1995.

[29] Royer J. Dessin du bonhomme : la personnalité de l'enfant dans tous ses états. Paris: Le Journal des psychologues; 2011.

[30] Scortegagna SA, de Villemor-Amaral AE. The Use of the Rorschach Method in the Investigation of Sexual Abuse of Children. Paideia 2012;22:261-69.

[31] Tibon S, Rothschild L, Appel L, Zeligman R. Assessing Effects of National Trauma on Adaptive Functioning of Mentally Healthy Adults: An Exploratory Rorschach Study. Psychology 2011;2:953-60.

\section{Annexe : texte du conte de la fourmi}

Un garçon (ou une petite fille) s'était un jour endormi dans l'herbe d'un pré. Voici une petite fourmi qui arrive et qui dit ; "Qu'est-ce que c'est que ça ? " Et comme elle était très curieuse elle se met à grimper sur le corps d'un petit garçon (ou de la petite fille).

1. Par quel endroit du corps de l'enfant arrive-t-elle, cette petite fourmi ?

2. De là, elle va se promener partout. Dis-moi tout ce qu'elle voi... Et puis...

3. Alors la fourmi a vu un petit trou, et elle a eu envie de voir ce qu'il y avait dedans.

- Quel était ce trou? Alors, elle entre et se promène partout à l'intérieur du corps de l'enfant.

- Qu'est-ce qu'elle voit? Et puis...

Alors, à la fin, elle ressort... 
- Par où ?

4. La petite fourmi a-t-elle trouvé que dans ses voyages elle a vu des choses belles ou pas belles ?

- Qu'est-ce qui était beau?

- Et pas beau?

5. Qu'a ressenti l'enfant pendant les voyages de la petite fourmi?

- Ça lui a fait plaisir ou mal ?

- Où?

6. Comment cette histoire s'est-elle terminée ? 